\title{
A Preconditioning Technique for Indefinite Systems Resulting from Mixed Approximations of Elliptic Problems*
}

\author{
By James H. Bramble and Joseph E. Pasciak
}

\begin{abstract}
This paper provides a preconditioned iterative technique for the solution of saddle point problems. These problems typically arise in the numerical approximation of partial differential equations by Lagrange multiplier techniques and/or mixed methods. The saddle point problem is reformulated as a symmetric positive definite system, which is then solved by conjugate gradient iteration. Applications to the equations of elasticity and Stokes are discussed and the results of numerical experiments are given.
\end{abstract}

1. Introduction. This paper provides and analyzes some new methods for the iterative solution of the algebraic systems corresponding to saddle point problems. Such systems typically arise in 'multiplier' or mixed discretizations of partial differential equations. Important examples of saddle point problems include the systems of discrete equations which result from the approximation of the equations of elasticity and Stokes [13], [14], [16]. Other examples result from the Lagrange multiplier and mixed formulations of second-order elliptic problems [2], [4], [5], [20]. Applications to mixed formulations for second-order problems will be described in a subsequent paper.

The elasticity/Stokes equations are perhaps the most important applications for the iterative methods to be described. Accordingly, we shall consider this application in detail. In addition, we shall include numerical examples illustrating the performance of the iterative method for these problems.

We shall develop the iterative methods in a general saddle point framework. Specifically, we consider systems of the form

$$
\left(\begin{array}{cc}
A & B^{*} \\
B & -C
\end{array}\right)\left(\begin{array}{l}
X \\
Y
\end{array}\right)=\left(\begin{array}{l}
F \\
G
\end{array}\right) .
$$

Here $F \in S^{1}$ and $G \in S^{2}$ are given, $X \in S^{1}$ and $Y \in S^{2}$ are to be determined, and $S^{1}$ and $S^{2}$ are Hilbert spaces. We assume that the operator $A$ is positive definite, $C$ is positive semidefinite (it may be 0 ), and $B^{*}$ is the adjoint of $B$. In practice, the above operators are often the matrices which determine the nodal values of the discrete solution corresponding to a finite element approximation.

1980 Mathematics Subject Classification (1985 Revision). Primary 65N30; Secondary 65F10.

*This manuscript has been authored under contract number DE-AC02-76CH00016 with the U.S. Department of Energy. Accordingly, the U.S. Government retains a non-exclusive, royaltyfree license to publish or reproduce the published form of this contribution, or allow others to do so, for U.S. Government purposes. This work was also supported in part under the National Science Foundation Grant No. DMS84-05352 and under the Air Force Office of Scientific Research, Contract No. ISSA86-0026. 
A sufficient condition for the solvability of (1.1) is the so-called 'inf-sup' condition [1], [9]. Applying block Gaussian elimination to (1.1) shows that $Y$ is the solution of

$$
\left(C+B A^{-1} B^{*}\right) Y=B A^{-1} F-G .
$$

Equation (1.2) will be solvable if the symmetric system $B A^{-1} B^{*}$ is positive definite. A direct computation gives

$$
\begin{aligned}
\left(B A^{-1} B^{*} V, V\right) & =\left(A A^{-1} B^{*} V, A^{-1} B^{*} V\right)=\sup U \in S^{1} \frac{\left(A A^{-1} B^{*} V, U\right)^{2}}{(A U, U)} \\
& =\sup U \in S^{1} \frac{(V, B U)^{2}}{(A U, U)} .
\end{aligned}
$$

Here and in the remainder of the paper, $(\cdot, \cdot)$ will denote the inner product on either $S^{1}$ or $S^{2}$, as will be clear from the context. Thus (1.2) is solvable if there is a positive constant $c$ satisfying

$$
\sup U \in S^{1} \frac{(V, B U)^{2}}{(A U, U)} \geq c\|V\|^{2} \quad \text { for all } V \in S^{2},
$$

where $\|\cdot\|^{2}$ is the norm on $S^{2}$. Equation (1.4) is equivalent to the inf-sup condition.

If $(1.4)$ holds, then $\left(C+B A^{-1} B^{*}\right)$ is a symmetric positive definite system. One can then solve (1.2) by applying any of the standard iterative methods for positive definite systems, for example, the conjugate gradient method or a simple linear iteration. Then, once $Y$ is known, $X$ is given by

$$
X=A^{-1}\left(F-B^{*} Y\right) \text {. }
$$

We note here that the well-known Uzawa Algorithm is a linear iteration technique applied to the solution of (1.2). In the case of the discretizations of the equations of elasticity and Stokes by mixed methods [16], the convergence rate of these iterative methods is usually independent of the number of unknowns in the discrete system. Each step of the iteration requires the evaluation of the operator $\left(C+B A^{-1} B^{*}\right)$ on some residual vector.

One drawback of the above solution processes (and, in particular, the Uzawa Algorithm) is that the action of $A^{-1}$ must be computed on various vectors. In many important applications, the cost of computing the action of $A^{-1}$ may be much more than the cost of evaluating the action of some other symmetric positive definite operator $A_{0}^{-1}$ which is a preconditioner for $A$. One is then faced with the question of how to effectively use the preconditioner $A_{0}^{-1}$ to obtain the solution to (1.1). Naively, one could use a double iteration, i.e., use a conjugate gradient iteration for (1.2) as an outer iterative scheme and use a preconditioned conjugate gradient iteration to evaluate $A^{-1}$. The problem with this approach is that in order to ensure convergence of the outer iteration, it becomes necessary to iterate the inner until it converges within computer roundoff, thus making the overall solution process somewhat costly.

In this paper, we will develop a single-level iteration approach for the solution of (1.1) which utilizes $A_{0}^{-1}$ and does not require the computation of the action of $A^{-1}$. We develop a positive definite reformulation of (1.1) and iterate for its solution. In many applications, the amount of work required to solve (1.1) with this single-level 
iteration is comparable to that required for one evaluation of $A^{-1}$ in the double iteration.

We should remark that in the case of the equations of elasticity and Stokes with the boundary conditions considered in this paper, the operator $A$ is equivalent to two copies of a second-order elliptic operator in one variable. Thus, with the iterative techniques to be developed, the problem of efficiently solving the discrete Stokes systems reduces to the problem of preconditioning the discrete systems corresponding to second-order elliptic equations. Preconditioners for second-order equations have been a topic of intensive theoretical and computational research and many techniques have appeared in the literature ([3], [7], [8], [10] and the included references).

It is possible to use the preconditioner $A_{0}^{-1}$ in an Arrow-Hurwicz [23] 'like' algorithm to obtain the solution of (1.1). This Arrow-Hurwicz algorithm requires the selection of iteration parameters. In contrast, the application of the conjugate gradient method with the reformulation to be developed in this paper does not require any parameters and leads to an 'optimally' converging scheme.

The outline of the remainder of the paper is as follows. In Section 2 we reformulate the saddle point problem as a symmetric positive definite system, and a general comparison theorem is given for the reformulated system. In Section 3 we describe the application of this technique to the equations of elasticity and Stokes. We consider applications to the Lagrange multiplier formulation of Dirichlet's problem in Section 4. Finally, the results of numerical experiments illustrating the iterative method's performance are given in Section 5.

2. The Positive Definite Reformulation. In this section we shall describe a way to develop a positive definite system for solving (1.1). The evaluation of this system and the implementation of the corresponding iterative method does not require the evaluation of $A^{-1}$ and takes full advantage of available preconditioners for $A$. We prove a comparison theorem which can be used to estimate the condition number of this system. In the applications described in later sections, this theorem can be used to show that the new systems will be either well conditioned or easily preconditioned.

Let $A_{0}$ be a preconditioner for $A$. To be effective, $A_{0}$ should satisfy two criteria. Firstly, the cost of applying the inverse of $A_{0}$ on a vector should be considerably less than the cost of applying $A^{-1}$. The second condition required for the effectiveness of $A_{0}$ is that there exist positive constants $\alpha_{0}$ and $\alpha_{1}$ whose ratio $\alpha_{1} / \alpha_{0}$ is small (preferably bounded independently of the dimension of $S^{1}$ ) such that

$$
\alpha_{0}(A U, U) \leq\left(A_{0} U, U\right) \leq \alpha_{1}(A U, U) \text { for all } U \in S^{1} .
$$

We shall make the additional assumption that

$$
\alpha_{1}<1
$$

Note that for $\alpha=1-\alpha_{0}$,

$$
0<\left(\left(A-A_{0}\right) U, U\right) \leq \alpha(A U, U)
$$

holds for all $U \neq 0$ in $S^{1}$. 
To derive the positive definite system, we again apply row operations to the matrix (1.1). Straightforward manipulations give

$$
M\left(\begin{array}{l}
X \\
Y
\end{array}\right) \equiv\left(\begin{array}{cc}
A_{0}^{-1} A & A_{0}^{-1} B^{*} \\
B A_{0}^{-1}\left(A-A_{0}\right) & C+B A_{0}^{-1} B^{*}
\end{array}\right)\left(\begin{array}{l}
X \\
Y
\end{array}\right)=\left(\begin{array}{c}
A_{0}^{-1} F \\
B A_{0}^{-1} F-G
\end{array}\right)
$$

As a consequence of (2.3), we can define the following inner product on $S^{1} \times S^{2}$,

$$
\left[\left(\begin{array}{l}
U \\
V
\end{array}\right),\left(\begin{array}{c}
W \\
X
\end{array}\right)\right] \equiv(A U, W)-\left(A_{0} U, W\right)+(V, X) \text {. }
$$

We note that

$$
\begin{aligned}
{\left[M\left(\begin{array}{l}
U \\
V
\end{array}\right),\left(\begin{array}{c}
W \\
X
\end{array}\right)\right]=} & \left(\left(A A_{0}^{-1} A-A\right) U, W\right)+\left(\left(A-A_{0}\right) A_{0}^{-1} B^{*} V, W\right) \\
& +\left(B A_{0}^{-1}\left(A-A_{0}\right) U, X\right)+\left(\left(C+B A_{0}^{-1} B^{*}\right) V, X\right),
\end{aligned}
$$

and hence the operator $M$ is symmetric in the inner product defined by (2.5). We will show (see Theorem 1) that $M$ is also positive definite. Thus we can apply any of a number of iterative techniques [19], [24] to solve (2.4). In the Appendix, we include a discussion of the application of the conjugate gradient method in the inner product (2.5) for the solution of (2.4).

Remark 1. In applications, a preconditioner is usually selected which a priori may not satisfy (2.2). It is easy to compute an appropriate scaling factor for the preconditioner so that the scaled preconditioner satisfies (2.2). This can be done, for example, by using the power method to estimate the lowest eigenvalue of $A_{0}^{-1} A$ with the unscaled operator $A_{0}^{-1}$. The cost of this computation is small compared to the cost of the subsequent iteration for the solution of (2.4). Note that the computation of the action of the inverse of the scaled operator is no more difficult than that corresponding to the unscaled operator, and the comparison ratio $\alpha_{1} / \alpha_{0}$ remains unchanged.

The following result provides a basic conditioning estimate for the operator $M$.

THEOREM 1. Let $\tilde{M}$ be given by

$$
\tilde{M} \equiv\left(\begin{array}{lc}
I & 0 \\
0 & C+B A^{-1} B^{*}
\end{array}\right) .
$$

Then the following inequalities hold:

$$
\lambda_{0}\left[\tilde{M}\left(\begin{array}{l}
U \\
V
\end{array}\right),\left(\begin{array}{l}
U \\
V
\end{array}\right)\right] \leq\left[M\left(\begin{array}{l}
U \\
V
\end{array}\right),\left(\begin{array}{l}
U \\
V
\end{array}\right)\right] \leq \lambda_{1}\left[\tilde{M}\left(\begin{array}{l}
U \\
V
\end{array}\right),\left(\begin{array}{l}
U \\
V
\end{array}\right)\right],
$$

where

$$
\lambda_{0}=\left(1+\frac{\alpha}{2}+\sqrt{\alpha+\frac{\alpha^{2}}{4}}\right)^{-1} \quad \text { and } \quad \lambda_{1}=\frac{1+\sqrt{\alpha}}{1-\alpha} .
$$

Remark 2. In the case of the Stokes equation, $\tilde{M}$ is well conditioned and hence the theorem implies that $M$ is also. In other applications, the theorem is used to show that any preconditioner for $\tilde{M}$ can be used as a preconditioner for $M$. Thus, the problem of constructing a preconditioner for $M$ is reduced to that of constructing one for (1.2).

Remark 3. The constants appearing in the inequalities of (2.8) tend to one as $\alpha$ tends to zero. This means that as the preconditioner tends to $A$ in an appropriate 
way, the eigenvalues of $\tilde{M}^{-1} M$ tend uniformly to one. Thus, it is advantageous to scale the preconditioner so that (2.3) holds with $\alpha$ as small as possible. This is equivalent to scaling the preconditioner so that $\alpha_{1}$ is close to 1 .

Remark 4. The theorem also gives some indication of how well the iterative scheme behaves when the condition number $K$ of $A_{0}^{-1} A$ becomes large. In this case, if $A_{0}$ is appropriately scaled, $(1-\alpha)^{-1}$ grows like $K$. The theorem guarantees that the condition number for $\tilde{M}^{-1} M$ grows at most proportional to $K$.

Proof. To prove the theorem, we shall use an appropriate decomposition in $S^{1} \times S^{2}$. Let $(U, V) \in S^{1} \times S^{2}$, and write

$$
\left(\begin{array}{c}
U \\
V
\end{array}\right)=\left(\begin{array}{c}
U_{0} \\
0
\end{array}\right)+\left(\begin{array}{c}
U_{H} \\
V
\end{array}\right)
$$

where $U_{H}$ is the unique function in $S^{1}$ satisfying

$$
A U_{H}+B^{*} V=0 .
$$

It is then straightforward to verify that

$$
\begin{aligned}
\left(A U_{H}, U_{H}\right) & =\left(B A^{-1} B^{*} V, V\right), \\
{\left[M\left(\begin{array}{c}
U_{H} \\
V
\end{array}\right),\left(\begin{array}{c}
U_{H} \\
V
\end{array}\right)\right] } & =\left(B A^{-1} B^{*} V, V\right)+(C V, V),
\end{aligned}
$$

and

$$
\left[M\left(\begin{array}{c}
U \\
V
\end{array}\right),\left(\begin{array}{c}
U \\
V
\end{array}\right)\right]=\left[M\left(\begin{array}{c}
U_{0} \\
0
\end{array}\right),\left(\begin{array}{c}
U_{0} \\
0
\end{array}\right)\right]+\left[M\left(\begin{array}{c}
U_{H} \\
V
\end{array}\right),\left(\begin{array}{c}
U_{H} \\
V
\end{array}\right)\right] .
$$

We start by proving the first inequality of (2.8). By definition,

$$
\left[\tilde{M}\left(\begin{array}{l}
U \\
V
\end{array}\right),\left(\begin{array}{l}
U \\
V
\end{array}\right)\right]=\left(\left(A-A_{0}\right) U, U\right)+\left(\left(C+B A^{-1} B^{*}\right) V, V\right) .
$$

Thus,

$$
\begin{aligned}
{\left[\tilde{M}\left(\begin{array}{l}
U \\
V
\end{array}\right),\left(\begin{array}{l}
U \\
V
\end{array}\right)\right] \leq } & (1+\gamma)\left(\left(A-A_{0}\right) U_{0}, U_{0}\right) \\
& +\left(1+\gamma^{-1}\right)\left(\left(A-A_{0}\right) U_{H}, U_{H}\right) \\
& +\left(B A^{-1} B^{*} V, V\right)+(C V, V)
\end{aligned}
$$

holds for any positive $\gamma$. Combining (2.3) and (i) gives

$$
\left(\left(A-A_{0}\right) U_{H}, U_{H}\right) \leq \alpha\left(B A^{-1} B^{*} V, V\right) .
$$

Hence,

$$
\begin{aligned}
{\left[\tilde{M}\left(\begin{array}{l}
U \\
V
\end{array}\right),\left(\begin{array}{l}
U \\
V
\end{array}\right)\right] \leq } & (1+\gamma)\left(\left(A-A_{0}\right) U_{0}, U_{0}\right) \\
& +\left(1+\left(1+\gamma^{-1}\right) \alpha\right)\left(B A^{-1} B^{*} V, V\right)+(C V, V) .
\end{aligned}
$$

But

$$
\left[M\left(\begin{array}{c}
U_{0} \\
0
\end{array}\right),\left(\begin{array}{c}
U_{0} \\
0
\end{array}\right)\right]=\left(\left(A-A_{0}\right) A_{0}^{-1}\left(A-A_{0}\right) U_{0}, U_{0}\right)+\left(\left(A-A_{0}\right) U_{0}, U_{0}\right)
$$


Combining (2.11), (2.12), and (ii) gives

$$
\begin{aligned}
{\left[\tilde{M}\left(\begin{array}{l}
U \\
V
\end{array}\right),\left(\begin{array}{c}
U \\
V
\end{array}\right)\right] \leq } & (1+\gamma)\left[M\left(\begin{array}{c}
U_{0} \\
0
\end{array}\right),\left(\begin{array}{c}
U_{0} \\
0
\end{array}\right)\right] \\
& +\left(1+\left(1+\gamma^{-1}\right) \alpha\right)\left[M\left(\begin{array}{c}
U_{H} \\
V
\end{array}\right),\left(\begin{array}{c}
U_{H} \\
V
\end{array}\right)\right] .
\end{aligned}
$$

The first inequality of (2.8) follows from (iii) and (2.13) with

$$
\gamma=\frac{\alpha}{2}+\sqrt{\alpha+\frac{\alpha^{2}}{4}}
$$

We next prove the second inequality of (2.8). We shall consider the two terms of (iii) separately. For the first term, by (2.1) and (2.3), it follows that

$$
\left(A_{0}^{-1} W, W\right) \leq \frac{\alpha}{1-\alpha}\left(\left(A-A_{0}\right)^{-1} W, W\right) .
$$

Using (2.12) and (2.14) yields

$$
\begin{aligned}
{\left[M\left(\begin{array}{c}
U_{0} \\
0
\end{array}\right),\left(\begin{array}{c}
U_{0} \\
0
\end{array}\right)\right] } & \leq(1-\alpha)^{-1}\left(\left(A-A_{0}\right) U_{0}, U_{0}\right) \\
\leq(1-\alpha)^{-1}\left\{(1+\gamma)\left(\left(A-A_{0}\right) U, U\right)\right. & \left.+\left(1+\gamma^{-1}\right)\left(\left(A-A_{0}\right) U_{H}, U_{H}\right)\right\} .
\end{aligned}
$$

By (2.10), (ii), (iii) and (2.15),

$$
\begin{aligned}
{\left[M\left(\begin{array}{l}
U \\
V
\end{array}\right),\left(\begin{array}{l}
U \\
V
\end{array}\right)\right] \leq } & \frac{1+\gamma}{1-\alpha}\left(\left(A-A_{0}\right) U, U\right) \\
& +\left(1+\frac{\alpha\left(1+\gamma^{-1}\right)}{1-\alpha}\right)\left(\left(B A^{-1} B^{*} V, V\right)+(C V, V)\right) .
\end{aligned}
$$

The second inequality of (2.8) follows from taking $\gamma=\sqrt{\alpha}$ in (2.16).

To see that the bounds in Theorem 1 are essentially sharp, we consider the following example. Let $A$ be a symmetric positive definite operator on $R^{n}$. We apply the reformulation of Section 2 to the system

$$
\left(\begin{array}{cc}
A & A^{1 / 2} \\
A^{1 / 2} & 0
\end{array}\right)\left(\begin{array}{l}
X \\
Y
\end{array}\right)=\left(\begin{array}{l}
F \\
G
\end{array}\right)
$$

with $A_{0}=(1-\alpha) A$ and $0<\alpha<1$. The reformulated system $M$ is given by

$$
M=(1-\alpha)^{-1}\left(\begin{array}{cc}
I & A^{-1 / 2} \\
\alpha A^{1 / 2} & I
\end{array}\right) .
$$

For this example, $\tilde{M}$ is the identity. It is easy to show that $M$ has only the two eigenvalues $\gamma_{0}=(1-\sqrt{\alpha}) /(1-\alpha)$ and $\gamma_{1}=(1+\sqrt{\alpha}) /(1-\alpha)$. Thus the estimate for $\lambda_{1}$ in the theorem is sharp. The estimate for $\lambda_{0}$ in the theorem is essentially sharp in that

(i) both $\lambda_{0}$ and $\gamma_{0}$ remain bounded away from zero for $0<\alpha<1$, and

(ii) both $1-\lambda_{0}$ and $1-\gamma_{0}$ tend to zero like $\sqrt{\alpha}$ as $\alpha$ tends to zero.

3. The Elasticity - Stokes Application. In this section we shall apply the reformulation developed in Section 2 to the steady state Stokes equations and the equations of linear elasticity. Let $\Omega$ be a domain in $N$-dimensional Euclidean space 
for $N=2$ or $N=3$. The velocity-pressure formulation of the steady state Stokes problem is: Find $\mathbf{u}$ and $p$ satisfying

$$
\begin{gathered}
-\sum_{j=1}^{N} \frac{\partial}{\partial x_{j}} \varepsilon_{i j}(\mathbf{u})+\frac{\partial p}{\partial x_{i}}=F_{i} \quad \text { in } \Omega, \\
\nabla \cdot \mathbf{u}=0 \quad \text { in } \Omega \\
\sum_{j=1}^{N} \varepsilon_{i j}(\mathbf{u}) n_{j}-p n_{i}=0 \quad \text { on } \Gamma_{1} \\
\mathbf{u}=0 \quad \text { on } \Gamma_{2}, \\
\int_{\Omega} p=0 \quad \text { if } \Gamma_{1}=\varnothing
\end{gathered}
$$

for $i=1, \ldots, N$. Here we have partitioned the boundary of $\Omega$ into $\Gamma_{1} \cup \Gamma_{2}$, and $\varepsilon_{i j}(\mathbf{u})$ is the usual symmetric strain tensor defined by

$$
\varepsilon_{i j}(\mathbf{u}) \equiv \frac{1}{2}\left\{\frac{\partial u_{i}}{\partial x_{j}}+\frac{\partial u_{j}}{\partial x_{i}}\right\}
$$

To get the equations of linear elasticity, we replace Eq. (3.2) by

$$
-\frac{1}{1-2 \nu} \nabla \cdot \mathbf{u}=p
$$

In Eq. (3.6), $0<\nu<1 / 2$ is Poisson's ratio. We note that the iterative methods to be described converge to the discrete solution with rates independent of $\nu$.

The two types of boundary conditions (3.3) and (3.4) are interesting in the case of linear elasticity. Condition (3.3) corresponds to a free boundary where the material is allowed to deform at the boundary. Condition (3.4) corresponds to a fixed boundary where the material is clamped. Two other types of boundary conditions are possible, but will not be treated in this paper.

We consider a weak formulation of problem (3.1)-(3.6). Let $(\cdot, \cdot)$ denote the $L^{2}(\Omega)$ inner product and $\|\cdot\|$ the corresponding norm. Let $H(\Omega)$ be the set of functions defined on $\Omega$ which vanish on $\Gamma_{2}$ and which along with their first derivatives are square integrable on $\Omega$. Define $\mathbf{H}(\Omega) \equiv H(\Omega) \times H(\Omega)$ and let $\|\cdot\|_{1}$ denote the corresponding norm. To simplify the presentation, we shall assume that $\Gamma_{2} \neq \varnothing$.** Let $\Pi=L^{2}(\Omega)$ if $\Gamma_{1} \neq \varnothing$ and $\Pi=L^{2}(\Omega) / R \equiv\left\{\phi \in L^{2}(\Omega) \mid \int_{\Omega} \phi d x=0\right\}$ if $\Gamma_{1}=\varnothing$. A weak formulation of (3.1)-(3.6) is then given by

$$
\bar{A}(\mathbf{u}, \mathbf{v})-(p, \nabla \cdot \mathbf{v})=(\mathbf{F}, \mathbf{v}) \quad \text { for all } \mathbf{v} \in \mathbf{H}(\Omega)
$$

and

$$
(\nabla \cdot \mathbf{u}, q)+\gamma(p, q)=0 \text { for all } q \in \Pi,
$$

where $\gamma=0$ in the Stokes case (3.2), and $\gamma=1-2 \nu$ in the elasticity case (3.6). Here the form $\bar{A}$ is defined by

$$
\bar{A}(\mathbf{w}, \mathbf{v}) \equiv \sum_{i, j=1}^{N} \int_{\Omega} \varepsilon_{i j}(\mathbf{w}) \varepsilon_{i j}(\mathbf{v}) d x .
$$

${ }^{* *}$ If $\Gamma_{2}=\varnothing$, then for existence of solutions to (3.1)-(3.6) compatibility assumptions must be made on $\mathbf{F}$. In such cases, solutions are determined only modulo a rigid motion. This results in some changes in the algorithms. 
We note that an alternative weak form may be derived in the special case when $\Gamma_{1}=\varnothing$. For example, we consider the Stokes application; the situation is completely analogous for the equations of linear elasticity. Equation (3.1) can be rewritten

$$
-\frac{1}{2} \Delta \mathbf{u}+\nabla p=\mathbf{F} \quad \text { in } \Omega
$$

Then for $\Gamma_{1}=\varnothing,(3.10)$ implies that $\mathbf{u}$ satisfies the alternative weak formulation

$$
\begin{aligned}
\frac{1}{2} \bar{D}(\mathbf{u}, \mathbf{v})-(p, \nabla \cdot \mathbf{v}) & =(\mathbf{F}, \mathbf{v}) \quad \text { for all } \mathbf{v} \in \mathbf{H}(\Omega) \\
(\nabla \cdot \mathbf{u}, q) & =0 \text { for all } q \in \Pi
\end{aligned}
$$

Here,

$$
\bar{D}(\mathbf{w}, \mathbf{v}) \equiv D\left(w_{1}, v_{1}\right)+D\left(w_{2}, v_{2}\right),
$$

and $D(\cdot, \cdot)$ is the Dirichlet form defined by

$$
D(v, w) \equiv \int_{\Omega} \nabla u \cdot \nabla v d x
$$

Computationally, formulation (3.11)-(3.12) is easier to handle than (3.7)-(3.8) since there are no cross terms between the components of $\mathbf{w}$ and $\mathbf{v}$ in the form $\bar{D}$.

To approximately solve (3.7)-(3.8) or (3.11)-(3.12), we introduce a pair of approximation subspaces $\mathbf{H}_{h}, \Pi_{h}$ indexed by $h$ in the interval $0<h<1$. Functions in $\mathbf{H}(\Omega)$ and $\Pi$ will be approximated by functions in $\mathbf{H}_{h}$ and $\Pi_{h}$, respectively. We will assume that the inf-sup condition holds for the pair of spaces; i.e., we assume that there is a constant $c$ which does not depend upon $h$ such that

$$
\inf Q \in \Pi_{h} \sup \mathbf{V} \in \mathbf{H}_{h} \frac{(Q, \nabla \cdot \mathbf{V})}{\|\mathbf{V}\|_{1}\|Q\|} \geq c \text {. }
$$

Many subspace pairs satisfying (3.14) have been studied, and their approximation properties are well known [16], [18], [21]. The bibliography of [18] contains additional references.

The approximations to the functions $\mathbf{u}, p$ are defined by replacing the spaces in (3.7)-(3.8) by their discrete counterparts. Specifically, the approximations are defined as the functions $\mathrm{U} \in \mathbf{H}_{h}$ and $P \in \Pi_{h}$ satisfying

$$
\bar{A}(\mathbf{U}, \mathbf{V})-(P, \nabla \cdot \mathbf{V})=(\mathbf{F}, \mathbf{V}) \text { for all } \mathbf{V} \in \mathbf{H}_{h}
$$

and

$$
(\nabla \cdot \mathbf{U}, Q)+\gamma(P, Q)=0 \text { for all } Q \in \Pi_{h} .
$$

The variables $U$ and $P$ satisfy a system of the form (1.1). To see this, we need only cast (3.15)-(3.16) in the notation of Section 1. First, we set $S^{1}=\mathbf{H}_{h}$ and $S^{2}=\Pi_{h}$. Define $A: S^{1} \mapsto S^{1}$ by $A \mathrm{~V} \equiv \mathrm{W}$, where $\mathrm{W}$ satisfies

$$
(\mathbf{W}, \mathbf{X})=\bar{A}(\mathbf{V}, \mathbf{X}) \text { for all } \mathbf{X} \in S^{\mathbf{1}} \text {. }
$$

Define $B: S^{1} \mapsto S^{2}$ by $B \mathrm{~V} \equiv Q$, where $Q$ satisfies

$$
(Q, R)=-(\nabla \cdot \mathrm{V}, R) \text { for all } R \in S^{2} \text {. }
$$


Finally, set $C$ to be $\gamma$ times the identity operator on $S^{2}$. Then for appropriate $F$,

$$
\left(\begin{array}{cc}
A & B^{*} \\
B & -C
\end{array}\right)\left(\begin{array}{c}
\mathbf{U} \\
P
\end{array}\right)=\left(\begin{array}{c}
F \\
0
\end{array}\right) \text {. }
$$

Hence, we can apply the reformulation (2.4) as well as Theorem 1 for the solution $\mathrm{U}, P$. We then have the following theorem.

THEOREM 2. Assume that a preconditioner $A_{0}^{-1}$ is given and satisfies (2.3) with constant $\alpha$. Let $M$ be given by (2.4) with $A, B$, and $C$ as above, and assume that (3.14) holds. Then the solution of (3.15)-(3.16) satisfies the system

$$
M\left(\begin{array}{c}
\mathrm{U} \\
P
\end{array}\right)=\left(\begin{array}{c}
A_{0}^{-1} F \\
B A_{0}^{-1} F
\end{array}\right) .
$$

and the condition number of $M$ is bounded by $\tilde{c}(1-\alpha)^{-1}$. Here $\tilde{c}$ is a positive constant which is independent of $h$.

Proof. We only need to estimate the condition number of $M$. By Theorem 1, it suffices to show that there are positive constants $c_{0}$ and $c_{1}$ not depending on $h$ satisfying

$$
c_{0}\|Q\|^{2} \leq\left(\left(C+B A^{-1} B^{*}\right) Q, Q\right) \leq c_{1}\|Q\|^{2} \quad \text { for all } Q \in \Pi_{h} .
$$

The form $\bar{A}(\cdot, \cdot)$ is equivalent to $\|\cdot\|_{1}^{2}$ on $\mathbf{H}_{h}$. We have by (1.3) and (3.14) that

$$
\begin{aligned}
\left(\left(C+B A^{-1} B^{*}\right) Q, Q\right) & =\gamma\|Q\|^{2}+\sup \mathbf{V} \in \mathbf{H}_{h} \frac{(Q, \nabla \cdot \mathbf{V})^{2}}{\bar{A}(\mathbf{V}, \mathbf{V})} \\
& \geq c_{0}\|Q\|^{2} .
\end{aligned}
$$

By (1.3) and the Schwarz inequality, it follows that

$$
\left(\left(C+B A^{-1} B^{*}\right) Q, Q\right) \leq\|Q\|^{2}\left(\gamma+\frac{\|\mathbf{V}\|_{1}^{2}}{\bar{A}(\mathbf{V}, \mathbf{V})}\right) \leq c_{1}\|Q\|^{2} .
$$

This completes the proof of the theorem.

Note that if $\alpha$ is independent of $h$, then the condition number of $M$ is bounded independently of $h$. This holds when $A_{0}$ is such that (2.1) is satisfied with $\alpha_{0}$ and $\alpha_{1}$ independent of $h$.

4. The Lagrange Multiplier Method for Dirichlet's Problem. In this section we apply the reformulation of Section 2 to Lagrange multiplier type methods for the numerical approximation of second-order elliptic equations. We will consider in detail the Lagrange multiplier method for Dirichlet's problem as developed in [2], [4]. In this case, we shall see that the resulting operator $\tilde{M}$ of Theorem 1 will not be uniformly well conditioned (independent of $h$ ). In a similar way, the techniques of Section 2 could be applied to the discrete systems developed in [5] for magnetostatic field problems. However, a complete description of this application is somewhat involved and will not be given.

We shall consider the problem of approximating the solution $u$ satisfying

$$
\begin{aligned}
L u=f & \text { in } \Omega \\
u=g & \text { on } \partial \Omega,
\end{aligned}
$$


where $\Omega$ is a bounded domain in $R^{N}$ with smooth boundary. Here $L$ is given by

$$
L u=-\sum_{i, j=1}^{N} \frac{\partial}{\partial x_{i}}\left(a_{i j} \frac{\partial u}{\partial x_{j}}\right)+a u .
$$

The assumptions on $a$ and $a_{i j}$ are as in [4].

To define the Lagrange multiplier approximation to (4.1), we shall need to introduce two sets of finite element approximation subspaces. The first set $\left\{S_{h}, 0<h<\right.$ $1\}$ consists of approximation spaces defined on $\Omega$. The second set $\left\{\dot{S}_{k}, 0<k<1\right\}$ consists of approximation spaces defined on $\partial \Omega$. We shall not give explicit assumptions and descriptions of these spaces; these details are available in [4]. Let us, however, note that functions in $S_{h}$ are not required to satisfy essential boundary conditions and that $k$ must be appropriately related to $h$.

We shall also use a generalized Dirichlet integral $A_{\beta}(\cdot, \cdot)$ defined by

$$
A_{\beta}(v, w)=\sum_{i, j=1}^{N} \int_{\Omega} a_{i j} \frac{\partial v}{\partial x_{i}} \frac{\partial w}{\partial x_{j}} d x+(a v, w)+\beta\langle v, w\rangle
$$

Here, $\beta$ is a nonnegative constant, $(\cdot, \cdot)$ denotes the $L^{2}$ inner product on $\Omega$, and $\langle\cdot, \cdot\rangle$ denotes the $L^{2}$ inner product on $\partial \Omega$.

We next define some discrete operators. As in [4], let $T_{h}: H^{-1}(\Omega) \mapsto S_{h}$ and $G_{h}: H^{-1 / 2}(\partial \Omega) \mapsto S_{h}$ be defined by

$$
A_{\beta}\left(T_{h} \theta, V\right)=(\theta, V) \text { for all } V \in S_{h},
$$

and

$$
A_{\beta}\left(G_{h} \sigma, V\right)=\langle\sigma, V\rangle \text { for all } V \in S_{h} .
$$

The Lagrange multiplier approximation to the solution $u$ of (4.1) is the function $U_{h} \in S_{h}$ defined by

$$
U_{h}=G_{h} \sigma_{k}+T_{h} f
$$

where $\sigma_{k}$ is the function in $\dot{S}_{k}$ which satisfies

$$
P_{k} G_{h} \sigma_{k}=P_{k}\left(g-T_{h} f\right) \text {. }
$$

Here, $P_{k}$ is defined to be the $L^{2}$ projection onto $\dot{S}_{k}$.

We shall see that $U_{h}$ and $\sigma_{k}$ satisfy a system of the form (1.1). First, we set $S^{1}=S_{h}$ and $S^{2}=\dot{S}_{k}$. We next define the operator $A: H^{1}(\Omega) \mapsto S_{h}$ by

$$
(A v, W)=A_{\beta}(v, W) \text { for all } W \in S_{h} .
$$

Straightforward manipulation of (4.2) and (4.3) gives

$$
\left(\begin{array}{cc}
A & -A G_{h} \\
-P_{k} & 0
\end{array}\right)\left(\begin{array}{c}
U_{h} \\
\sigma_{k}
\end{array}\right)=\left(\begin{array}{c}
f \\
-P_{k} g
\end{array}\right)
$$

We then set $B=-P_{k}$ and $C=0$. The matrix in (4.4) has the form of (1.1) since

$$
\left\langle P_{k} W, \delta\right\rangle=A_{\beta}\left(G_{h} \delta, W\right)=\left(A G_{h} \delta, W\right)
$$

Note that (4.3) can be derived from (4.4) in exactly the same way that (1.2) was derived from (1.1). 
Reference [4] discusses an algorithm for computing $U_{h}$ when one is willing to invert the $A_{\beta}(\cdot, \cdot)$ form , i.e., evaluate the action of $T_{h}$ and $G_{h}$. In that algorithm, a preconditioned iteration is set up for computing $\sigma_{k}$, the solution of (4.3). It is shown in [4] that the form $\left\langle G_{h} \sigma, \sigma\right\rangle$, when restricted to $\dot{S}_{k}$, is equivalent to the square of the norm of $\sigma$ in $H^{-1 / 2}(\partial \Omega)$. Accordingly, the operator $P_{k} G_{h}$ exhibits a condition number growth like $O\left(h^{-1}\right)$ and should be preconditioned. Techniques for constructing efficient preconditioners $\tilde{l}$ for $P_{k} G_{h}$ are discussed in [4] and [5]. For these preconditioners, there exist positive constants $c_{0}$ and $c_{1}$ independent of $h$ and $k$ satisfying

$$
c_{0}\langle\tilde{l} \delta, \delta\rangle \leq\left\langle G_{h} \delta, \delta\right\rangle \leq c_{1}\langle\tilde{l} \delta, \delta\rangle \quad \text { for all } \delta \in \dot{S}_{k} .
$$

It is often much more economical to compute the action of a preconditioner $A_{0}^{-1}$ than to evaluate the operators $T_{h}$ and $G_{h}$. In this case, we can use the technique of Section 2 to derive a well-conditioned iteration. The following theorem is a consequence of Theorem 1 and (4.6).

THEOREM 3. Assume that a preconditioner $A_{0}^{-1}$ has been given which satisfies (2.3). Let $M$ be given by (2.4) with $A, B$, and $C$ as above. Then the solution of (4.2)-(4.3) satisfies

$$
M\left(\begin{array}{c}
U_{h} \\
\sigma_{k}
\end{array}\right)=\left(\begin{array}{c}
A_{0}^{-1} f \\
P_{k}\left(g-A_{0}^{-1} f\right)
\end{array}\right) .
$$

If (4.6) holds, then there are positive constants $\beta_{0}$ and $\beta_{1}$ not depending on $h$ such that

$$
\beta_{0}\left[\tilde{J}\left(\begin{array}{c}
W \\
\delta
\end{array}\right),\left(\begin{array}{c}
W \\
\delta
\end{array}\right)\right] \leq\left[M\left(\begin{array}{c}
W \\
\delta
\end{array}\right),\left(\begin{array}{c}
W \\
\delta
\end{array}\right)\right] \leq \frac{\beta_{1}}{1-\alpha}\left[\tilde{J}\left(\begin{array}{c}
W \\
\delta
\end{array}\right),\left(\begin{array}{c}
W \\
\delta
\end{array}\right)\right]
$$

where

$$
\tilde{J} \equiv\left(\begin{array}{cc}
I & 0 \\
0 & \tilde{l}
\end{array}\right)
$$

Theorem 3 shows that the system $\tilde{J}^{-1} M$ is uniformly well conditioned if $\alpha$ is bounded away from one. Thus, preconditioned iteration for the solution of (4.7), with preconditioner $\tilde{J}^{-1}$, will converge rapidly. To apply such a scheme, we must evaluate the action of $M$. Suppose the operator $A_{0}: H^{1}(\Omega) \mapsto S_{h}$ is defined using another form $\tilde{A}$ by

$$
\left(A_{0} V, \theta\right)=\tilde{A}(V, \theta) \text { for all } \theta \in S_{h} .
$$

To apply $M$ to a vector $(V, q)^{t}$, i.e., compute

$$
\left(\begin{array}{c}
W \\
r
\end{array}\right)=M\left(\begin{array}{c}
V \\
q
\end{array}\right)
$$

we first compute $W=A_{0}^{-1}\left(A V+B^{*} q\right)$ and then $r=P_{k}(W-V)$. It is not difficult to see that $W$ solves

$$
\left(A_{0} W, \theta\right)=A_{\beta}(v, \theta)+\langle q, \theta\rangle \quad \text { for all } \theta \in S_{h} .
$$

Thus, neither the action of $T_{h}$ nor $G_{h}$ is required for the computation.

In an analogous manner, we can apply the reformulation of Section 2 to the discrete equations resulting from mixed methods for second-order elliptic equations, using, for example, the Raviart-Thomas elements [20]. The resulting operator $\tilde{M}$ (and hence $M$ ) is not uniformly well conditioned. The derivation of effective preconditioners for $\tilde{M}$ will be considered in a subsequent paper. 
5. Numerical Examples. In this section we shall present some results of numerical experiments using the reformulation described in Section 2. All of our examples will be for problems discussed in Section 3. We shall use the conjugate gradient method applied to the symmetric positive definite systems corresponding to either (1.2) or (2.4).

We first define the subspace pair $\left\{\mathbf{H}_{h}, \Pi_{h}\right\}$. For simplicity of exposition, we shall only describe these spaces when $\Omega$ is the unit square. Generalizations to more complex domains are possible.

Let $n>0$ be given. We start by breaking the square into $2 n \times 2 n$ square shaped subregions and define $h=1 / 2 n$ (see Figure 5.1). Let $x_{i} \equiv i h$ and $y_{j}=j h$ for $i, j=1, \ldots, 2 n$. We partition the square subregions into pairs of triangles using one of the subregion's diagonals (for example, the diagonal going from the bottom right corner to the upper left corner). Let $H_{h}$ be the collection of functions which vanish on the boundary of the square and are piecewise linear and continuous on this triangulation. The subspace $\mathbf{H}_{h}$ is defined by $H_{h} \times H_{h}$.

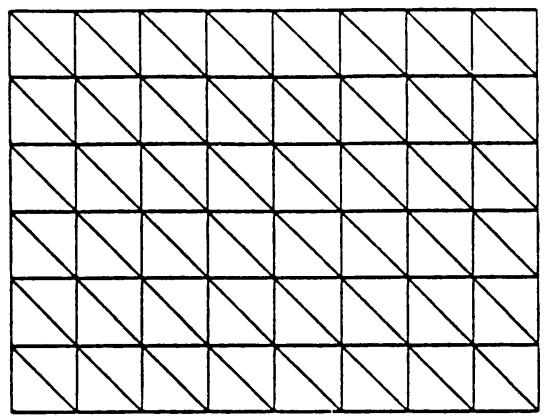

FIGURE 5.1

The triangular mesh.

To define the space $\Pi_{h}$, we first consider the space $\tilde{\Pi}_{h}$ which is defined to be the space of functions which are piecewise constant on the square regions (see Figure 5.2). It is interesting to note [17] that the subspace pair $\left\{\mathbf{H}_{h}, \tilde{\Pi}_{h}\right\}$ is not stable in $L^{2}$, i.e., the inequality

$$
\inf Q \in \tilde{\Pi}_{h} \sup V \in \mathbf{H}_{h} \frac{(Q, \nabla \cdot V)}{\|V\|_{H^{1}(\Omega)}\|Q\|_{L^{2}(\Omega)}} \geq c>0
$$

does not hold for $c$ independent of $h$. To get a stable subspace, we shall consider a somewhat smaller subspace of $\tilde{\Pi}_{h}$. Let $\theta_{k l}$ for $k, l=1, \ldots, 2 n$ be the function which is one on the subregion $\left[x_{k-1}, x_{k}\right] \times\left[y_{l-1}, y_{l}\right]$ and vanishes elsewhere. We define the functions $\phi_{i, j} \in \tilde{\Pi}_{h}$, for $i, j=1, \ldots, n$, by

$$
\phi_{i j} \equiv \theta_{2 i-1,2 j-1}-\theta_{2 i, 2 j-1}-\theta_{2 i-1,2 j}+\theta_{2 i, 2 j}
$$

We then define $\Pi_{h}$ by

$$
\Pi_{h} \equiv\left\{Q \in \tilde{\Pi}_{h} \mid\left(Q, \phi_{i j}\right)=0 \text { for } i, j=1, \ldots, n\right\} .
$$

An estimate of the form of (5.1) holds with $c$ independent of $h$ for the subspace pair $\left\{\mathbf{H}_{h}, \Pi_{h}\right\}$ [17]. Furthermore, the exclusion of the functions of the form (5.2) does 


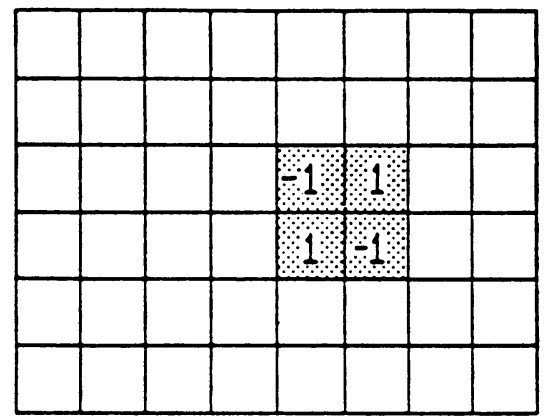

FIGURE 5.2

The rectangular mesh used for $\tilde{\Pi}_{h}$; the support (shaded) and values for a typical $\phi_{i j}$.

not result in a change in the order of approximation for the space (we obviously still have the subspace of constants on the mesh of size $2 h$ ).

Remark 5. The exclusion of functions of the form (5.2) poses no difficulty in practice. In fact, it only affects the definition of $B$ in a trivial way. By definition, $B \mathrm{~V} \equiv Q$, where $Q \in \Pi_{h}$ solves

$$
(Q, R)=-(\nabla \cdot \mathrm{V}, R) \text { for all } R \in \Pi_{h} .
$$

It is easy to see that $Q$ is the $L^{2}$ orthogonal projection of the function $\tilde{Q} \in \tilde{\Pi}_{h}$ satisfying

$$
(\tilde{Q}, R)=-(\nabla \cdot \mathrm{V}, R) \quad \text { for all } R \in \tilde{\Pi}_{h} .
$$

This projection is a trivial local operation since the supports of the functions $\left\{\phi_{i j}\right\}$ are disjoint. Furthermore, the computation of $\tilde{Q}$ is straightforward since the Gram matrix for (5.3) is diagonal (with the obvious choice of basis).

Remark 6. Other authors suggest solving for $U, P$ in the space $\mathbf{H}_{h}, \tilde{\Pi}_{h}[17]$. Then an accurate solution $U, \tilde{P}$ can be computed by projecting the resulting pressure $P$ into the space $\Pi_{h}$. This seems to be computationally more difficult than working in the smaller space $\Pi_{h}$ since the discrete system with $\tilde{\Pi}_{h}$ is not well conditioned, i.e., the constant $c$ in (5.1) grows like $h^{-2}$ [17].

Example 1. For our first example, we consider the Stokes equation

$$
\begin{gathered}
-\Delta \mathbf{u}+\nabla p=F \quad \text { in } \Omega, \\
\nabla \cdot \mathbf{u}=0 \quad \text { in } \Omega, \\
\mathbf{u}=0 \quad \text { on } \partial \Omega, \\
\int_{\Omega} p=0 .
\end{gathered}
$$

Since $\Gamma_{1}=\varnothing$, we can use the alternative weak formulation (3.11)-(3.12). The matrix $A$ then reduces to two copies of the discrete Laplace operator. In this case, there are many efficient methods for computing the action of $A^{-1}$ (see, for example, [22]) and hence we can apply either (1.2) or (2.4).

To investigate the efficiency of the iteration method of Section 2, we shall compare condition number and iteration results for the systems (1.2) and (2.4). For simplicity, we have taken $A_{0}$ to be $.8 A$ in (2.4). We give the condition numbers $K_{1}$ 
and $K_{2}$ corresponding to the systems resulting from (1.2) and (2.4), respectively, in Table 5.1. The condition number of the system ${ }^{* * *}$ can be used to provide bounds on the rate of iterative convergence. We also give the observed number of conjugate gradient iterations $N_{1}$ and $N_{2}$ necessary to reduce the discrete $L^{2}$ norm of the residual by a factor of .001 .

Note that in this simple example the use of the full system (i.e., that corresponding to (2.4)) is artificial since the action of $A^{-1}$ is no more difficult than the evaluation of $A_{0}^{-1}$. Later examples illustrate applications where (2.4) gives rise to algorithms which are more efficient since only preconditioners for $A$ need be evaluated.

TABLE 5.1

Convergence results for $(1.2)\left(K_{1}\right.$ and $\left.N_{1}\right)$ and (2.4) $\left(K_{2}\right.$ and $\left.N_{2}\right)$ applied to (5.4)

\begin{tabular}{|c|c|c|c|c|}
\hline$h$ & $K_{1}$ & $N_{1}$ & $K_{2}$ & $N_{2}$ \\
\hline $1 / 8$ & 4.5 & 6 & 9.0 & 11 \\
$1 / 16$ & 4.9 & 7 & 9.5 & 11 \\
$1 / 32$ & 5.2 & 7 & 9.8 & 11 \\
$1 / 64$ & 5.2 & 7 & 9.9 & 11 \\
\hline
\end{tabular}

Example 2. We consider a variable-coefficient Stokes problem in this example. Although this example may also be somewhat artificial, it illustrates the behavior of the composite method (2.4) on a nontrivial problem. We consider the solution of the following "Stokes like" problem:

$$
\begin{gathered}
-\nabla \cdot \mu \nabla \mathbf{u}+\nabla p=F \quad \text { in } \Omega, \\
\nabla \cdot \mathbf{u}=0 \quad \text { in } \Omega, \\
\mathbf{u}=0 \quad \text { on } \partial \Omega, \\
\int_{\Omega} p=0,
\end{gathered}
$$

where $\mu(x, y)=1+x y+x^{2}-y^{2} / 2$. In this example, we use .5 times the discrete Laplace operator in each component as a preconditioner. This preconditioner can be 'fast solved' by, for example, Fourier techniques [22]. In contrast, if we were to use (1.2) to solve the discrete equations corresponding to (5.5), we would have to solve variable coefficient discrete Dirichlet problems on $\Omega$. Although many techniques exist for the solution of such problems [12], [15], much more complicated and less efficient algorithms must be introduced. Table 5.2 gives convergence results for this example.

***As is well known [19], the rate of iterative convergence for conjugate gradient applied to the solution of the problem $M x=b$ can be bounded in terms of the condition number $K$ of $M$ by the expression $\left(M e_{i}, e_{i}\right) \leq 4 \rho^{2 i}\left(M e_{0}, e_{0}\right)$. Here $e_{i} \equiv x-x_{i},\left\{x_{i}\right\}$ is the sequence of iterates generated by the conjugate gradient algorithm, and $\rho \equiv(\sqrt{K}-1) /(\sqrt{K}+1)$. 
TABLE 5.2

Convergence results for Example 2.

\begin{tabular}{|c|c|c|}
\hline$h$ & $K$ & $N$ \\
\hline $1 / 8$ & 60 & 25 \\
$1 / 16$ & 74 & 28 \\
$1 / 32$ & 82 & 31 \\
$1 / 64$ & 97 & 31 \\
\hline
\end{tabular}

Example 3. In this example, we consider a Stokes equation with mixed boundary conditions. Specifically, we consider (3.1)-(3.4) with $\Omega$ the unit square and $\Gamma_{1}=$ $\{(x, y) \mid x=0$ or $x=1\}$. Accordingly, the functions in $H_{h}$ are only required to vanish on $\Gamma_{2}=\partial \Omega / \Gamma_{1}$. In addition, we must use the weak formulation (3.7)-(3.8), and $A$ becomes the 'stiffness matrix' derived from the form (3.9). Note that for this case, $A$ does not correspond to two component-wise copies of the discretization of an elliptic operator but contains cross terms between the components. However, Korn's inequality [11] gives

$$
c \bar{D}(V, V) \leq \bar{A}(V, V) \leq C \bar{D}(V, V) .
$$

Equation (5.6) implies that the $A$ of this example can be preconditioned by $A_{0}^{-1}$, where $A_{0}$ is the stiffness matrix corresponding to .5 times the form $\bar{D}(\cdot, \cdot)$ on $\mathbf{H}_{h} \times$ $\mathbf{H}_{h}$. The equations for computing $A_{0}^{-1} v$ decouple component-wise and can be solved using 'fast' direct methods. The condition number $K$ and the number of iterations $N$ are given in Table 5.3 as a function of $h$.

TABLE 5.3

Convergence results for Example 3.

\begin{tabular}{|c|c|c|}
\hline$h$ & $K$ & $N$ \\
\hline $1 / 8$ & 34 & 19 \\
$1 / 16$ & 39 & 20 \\
$1 / 32$ & 40 & 20 \\
$1 / 64$ & 40 & 20 \\
\hline
\end{tabular}

\section{Appendix}

For completeness, we include a discussion of the application of the conjugate gradient method to the solution of (2.4) in the inner product given by (2.5). We also include a remark which can be used to simplify these computations.

The conjugate gradient algorithm for the iterative solution of (2.4) in the inner product (2.5) is defined as follows. Let $Z_{0}$ be an initial approximation (for example, the zero vector) to the solution pair $(X, Y)^{t}$. Let

$$
\tilde{F} \equiv\left(\begin{array}{c}
A_{0}^{-1} F \\
B A_{0}^{-1} F-G
\end{array}\right)
$$


and define $P_{0} \equiv R_{0} \equiv \tilde{F}-M Z_{0}$. Then define

$$
\begin{aligned}
\alpha_{i} & =\frac{\left[R_{i}, P_{i}\right]}{\left[M P_{i}, P_{i}\right]}, \\
Z_{i+1} & =Z_{i}+\alpha_{i} P_{i}, \\
R_{i+1} & =\tilde{F}-M Z_{i+1}, \\
\beta_{i} & =\frac{\left[M R_{i+1}, P_{i}\right]}{\left[M P_{i}, P_{i}\right]}, \\
P_{i+1} & =R_{i+1}-\beta_{i} P_{i} .
\end{aligned}
$$

Remark 7. The evaluation of $A_{0}$ is not necessary for the implementation of the above algorithm, even though it appears implicitly in the definition of the inner product $[\cdot, \cdot]$. Note that the evaluation of $A_{0}$ is not necessary for the computation of $\left[M P_{i}, P_{i}\right]$ or $\left[M R_{i+1}, P_{i}\right]$ since the first component of either $M P_{i}$ or $M R_{i+1}$ is $A_{0}^{-1}$ applied to a known vector. The same holds for $\left[R_{i}, P_{i}\right]$. This observation is essential when using, for example, substructuring [6], [7], [8] or multigrid [3] preconditioners, where the action of the preconditioner $A_{0}^{-1}$ is defined as a process whose inverse is not easily computed.

Department of Mathematics

Cornell University

Ithaca, New York 14853

Brookhaven National Laboratory

Upton, New York 11973

1. A. K. AZIZ \& I. BABUŠKA, "Survey lectures on the mathematical foundations of the finite element method, Part I," in The Mathematical Foundations of the Finite Element Method with Applications to Partial Differential Equations (A.K. Aziz, ed.), Academic Press, New York, 1972, pp. 1-362.

2. I. BABUŠKA, "The finite element method with Lagrangian multipliers," Numer. Math., v. 20, 1973, pp. 179-192.

3. J. H. BRAMBLE, Iterative Methods for Solving Finite Element or Finite Difference Equations for Elliptic Problems, Lecture Notes. (Unpublished.)

4. J. H. BRAmble, "The Lagrange multiplier method for Dirichlet's problem," Math. Comp., v. 37, 1981, pp. 1-12.

5. J. H. BRAMBLE \& J. E. PASCIAK, "A boundary parametric approximation to the linearized scalar potential magnetostatic field problem," Appl. Numer. Math., v. 1, 1985, pp. 493-514.

6. J. H. BRAmble, J. E. PASCIAK \& A. H. SCHATZ, "An iterative method for elliptic problems on regions partitioned into substructures," Math. Comp., v. 46, 1986, pp. 361-369.

7. J. H. BRAmble, J. E. PASciaK \& A. H. SChatz, "The construction of preconditioners for elliptic problems by substructuring. I," Math. Comp., v. 47, 1986, pp. 103-134.

8. J. H. BRAmble, J. E. PASciak \& A. H. SChatz, "The construction of preconditioners for elliptic problems by substructuring. II," Math. Comp., v. 49, 1987, pp. 1-16.

9. F. BREZZI, "On the existence, uniqueness and approximation of saddle-point problems arising from Lagrange multipliers," RAIRO, 1974, pp. 129-151.

10. R. ChANDRA, Conjugate Gradient Methods for Partial Differential Equations, Yale Univ., Dept. of Comp. Sci., Rep. No. 129, 1978.

11. P. G. CiARLet, The Finite Element Method for Elliptic Problems, North-Holland, New York, 1978.

12. S. C. Eisenstat, M. C. GuRSKY, M. H. SChUltz \& A. H. Sherman, "Yale sparse matrix package, I. The symmetric codes," Internat. J. Numer. Methods Engrg., v. 18, 1982, pp. 1145-1151.

13. R. S. FALK, "An analysis of the finite element method using Lagrange multipliers for the stationary Stokes equations," Math. Comp., v. 30, 1976, pp. 241-269. 
14. R. S. FAlK \& J. E. OSBORN, "Error estimates for mixed methods," RAIRO Numer. Anal., v. 14, 1980, pp. 249-277.

15. A. GeORge \& J. W. LIU, Computer Solution of Large Sparse Positive Definite Systems, Prentice-Hall, Englewood Cliffs, N. J., 1981.

16. V. GIRAUlt \& P. A. RAVIART, Finite Element Approximation of the Navier-Stokes Equations, Lecture Notes in Math., vol. 749, Springer-Verlag, New York, 1981.

17. C. JOHNSON \& J. PITKÄRANTA, "Analysis of some mixed finite element methods related to reduced integration," Math. Comp., v. 38, 1982, pp. 375-400.

18. J. C. NEDELEC, "Elements finis mixtes incompressibles pour l'equation de Stokes dans $R^{3}$," Numer. Math., v. 39, 1982, pp. 97-112.

19. W. M. PATTERSON, 3RD, Iterative Methods for the Solution of a Linear Operator Equation in Hilbert Space - A Survey, Lecture Notes in Math., vol. 394, Springer-Verlag, New York, 1974.

20. P. A. RAVIART \& J. M. Thomas, "A mixed finite element method for 2-nd order elliptic problems," in Mathematical Aspects of Finite Element Methods (I. Galligani and E. Magenes, eds.), Lecture Notes in Math., vol. 606, Springer-Verlag, New York, 1977, pp. 292-315.

21. L. R. SCOTT \& M. Vogelius, Conforming Finite Element Methods for Incompressible and Nearly Incompressible Continua, Inst. for Phys. Sci. and Tech., Univ. of Maryland, Tech. Rep. BN-1018, 1984.

22. P. N. SWARZTRAUBER, "The methods of cyclic reduction, Fourier analysis and the FACR algorithm for the discrete solution of Poisson's equation on a rectangle," SIAM Rev., v. 19, 1977, pp. 490-501.

23. R. Temam, Navier-Stokes Equations, North-Holland, New York, 1977.

24. J. Westlake, A Handbook of Numerical Matrix Inversion and Solution of Linear Equations, Wiley, New York, 1968. 